\title{
Analysis of the Application Effect of the Modified Mews Score After Correction in Cardiovascular Medicine
}

\author{
Juxian $\mathrm{Wu}^{1}$, Lin $\mathrm{Li}^{2}$, Tong Zhong ${ }^{2}$, Lingyi Liu ${ }^{1}$, Xuelu Yang ${ }^{1}$, Xia Wei ${ }^{2}$, \\ ${ }^{1}$ Department of Cardionlogy, The People's Hospital of Zhuhai, Zhuhai, China \\ ${ }^{2}$ Department of Nursing, The People's Hospital of Zhuhai, Zhuhai, China
}

Email address:

zhweixia@126.com (Xia Wei)

${ }^{*}$ Corresponding author

\section{To cite this article:}

Juxian Wu, Lin Li, Tong Zhong, Lingyi Liu, Xuelu Yang, Xia Wei. Analysis of the Application Effect of the Modified Mews Score After Correction in Cardiovascular Medicine. European Journal of Preventive Medicine. Vol. 9, No. 3, 2021, pp. 79-82.

doi: 10.11648/j.ejpm.20210903.12

Received: April 26, 2021; Accepted: May 11, 2021; Published: May 15, 2021

\begin{abstract}
Objective: Compare the application effect of the modified MEWS score before and after correction in cardiovascular medicine. Methods: A retrospective collection of 322 patients who were hospitalized in the Cardiovascular Department of our hospital from June 2018 to June 2019 and met the inclusion and exclusion criteria were used as the control group; 347 patients who were hospitalized in the Department of Cardiovascular Medicine of our hospital from July 2019 to July 2020 and met the inclusion and exclusion criteria were collected as the experimental group. The experimental group used the modified MEWS score after adjustment, and the control group used the modified MEWS score. The clinical outcome and prediction accuracy of the two groups of patients were compared. Results: There was no difference in the clinical outcome of the two groups of patients $(P>0.05)$. The composition of different clinical outcomes of the two groups was different, and the composition ratio between the two groups of the same grade was also different. The prediction accuracy of the experimental group was significantly higher than that of the control group $(P<0.05)$. With death and ICU admission as the event endpoints, the correlation between the occurrence of the event and the MEWS score and the area under the ROC curve was statistically different between the two groups $(P<0.05)$. Conclusion: The corrected modified MEWS score further improves the accuracy of early warning based on the general modified MEWS score. It can be used to identify potentially critically ill patients.
\end{abstract}

Keywords: Cardiovascular Disease, MEWS Score, Clinical Outcome and Prognosis, Preventive Intervention Nursing

\section{Introduction}

AS a clinical department with a high incidence of acute and severe cardiovascular diseases such as heart failure and myocardial infarction, it can effectively reduce the incidence of adverse events and improve the prognosis of patients, through a standardized, timely, and effective way to observe the changes in the condition. Modified early warning score (MEWS) is a scoring tool developed by British experts, which is used to evaluate the situation and risk grading of patients in the emergency department or patients whose condition is not clear at the time of admission to achieve the purpose of pre-check triage $[1,2]$. Although MEWS has been widely used for clinical emergency evaluation [3-6], it lacks specialized indicators and can't make personalized risk prediction for patients in cardiovascular medicine $[7,8]$. The purpose of this study was to evaluate the predictive effect of the modified MEWS score after adjustment by comparing the application of the modified MEWS score before and after re-adjustment in cardiovascular medicine.

\section{Methods}

\subsection{Research Object}

A total of 322 patients who met the inclusion and exclusion criteria in the Department of Cardiovascular Medicine of our hospital from January 2019 to June 2019 were selected as the control group. The risk prediction was performed using the modified MEWS score. A total of 347 patients hospitalized in the same department from July 2019 to July 2020 were 
selected as the experimental group. The risk prediction was performed using the adjustment modified MEWS score. The control group ranged in age from 23 to 92 years old, with an average age of $62.3 \pm 15.1$ years old, including 176 male patients and 146 female patients. The experimental group ranged in age from 21 to 91 years old, with an average age of $61.8 \pm 14.6$ years old, including 193 male patients and 154 female patients. There was no statistical difference between the two groups of patients in general clinical data, and they were clinically comparable. This study has been reviewed and approved by the hospital ethics committee.

\subsection{Inclusion and Exclusion Criteria}

Inclusion criteria: (1) Age>18 years old; (2) Hospitalization time $>24$ hours. Exclusion criteria: (1) Patients who gave up treatment during hospitalization; (2) Patients with incomplete clinical data.

\subsection{Study Design}

The patients in the control group were evaluated for the severity of their illness from the evaluation of 5 indicators: heart rate, systolic blood pressure, respiratory frequency, body temperature, and consciousness. According to the scores, the patients were divided into mild and moderate groups (MEWS $<6$ points) and severe groups (MEWS 6 9 points), extremely severe groups (MEWS 6 9 points). The severity of the condition of the patients in the experimental group was evaluated from 8 indicators, including systolic blood pressure, diastolic blood pressure, respiratory rate, heart rate, blood oxygen saturation, arrhythmia, state of consciousness, and chest pain. According to the score, patients were divided into mild to moderate group (corrected $\mathrm{MEWS}<9$ points), severe group (corrected MEWS 9 16 points) and extremely severe group (corrected MEWS $\geq 16$ points). Follow up until the patient is discharged from the hospital or transferred to the ICU.

\subsection{Observation Indicators and Evaluation Criteria}

The clinical outcomes of the two groups of patients during hospitalization were compared. Clinical outcomes included: accident rate, rescue success rate, hospital stay, mortality rate, and ICU occupancy rate.

\subsection{Instrument}

This data has compiled with SPSS 21.0 for data analysis. Enumeration data were expressed by $\mathrm{X} \pm \mathrm{S}$ and t-test was used; measurement data were expressed by "\%", and chi-square test was used, and the Bonferroni adjustment method was used for pairwise comparison between groups. The Spearman rank correlation and ROC curve were used to compare the ability of MEWS before and after correction to identify the patients' death and admission to the ICU, and the best cut-off value was used to determine the warning level. " $P<0.05$ " was considered statistically different.

\section{Results}

\subsection{Comparison of the Clinical Outcome of the Two Groups of Patients}

There was no difference in the clinical outcome of the two groups of patients $(P>0.05)$, and there was no statistical difference between the two groups, which was clinically comparable. The specific values are shown in Table 1.

Table 1. Comparison of clinical outcomes between the two groups.

\begin{tabular}{|c|c|c|c|c|}
\hline Group & Control group $(n=322)$ & Experimental group $(n=347)$ & $t / X^{2}$ & $\boldsymbol{P}$ \\
\hline Accident (n,\%) & $287(89.1)$ & $296(85.3)$ & 2.18 & 0.14 \\
\hline Rescue (n,\%) & $175(54.0)$ & $201(57.9)$ & 1.02 & 0.31 \\
\hline Rescue success rate (n,\%) & $143(82.2)$ & $167(83.1)$ & 0.93 & 0.34 \\
\hline Hospital stay (d) & $5.35 \pm 1.22$ & $5.41 \pm 1.30$ & 0.61 & 0.54 \\
\hline Death $(\mathrm{n}, \%)$ & $29(9.0)$ & $31(8.9)$ & 0.01 & 0.92 \\
\hline ICU occupancy rate $(n, \%)$ & $133(41.3)$ & $159(45.8)$ & 1.39 & 0.24 \\
\hline
\end{tabular}

\subsection{The Composition of the Different Clinical Outcomes of the Two Groups of Patients}

The control group and the experimental group have a different compositions of different clinical outcomes. Pairwise comparisons between the groups show that the composition ratio between the two groups of the same grade is also different, and the prediction accuracy of the experimental group is significantly higher than that of the $(P<0.05)$. The specific values are shown in Table 2.

Table 2. The composition of the different clinical outcomes of the two groups of patients.

\begin{tabular}{|c|c|c|c|c|c|c|c|}
\hline \multirow{2}{*}{ Group } & \multicolumn{3}{|c|}{ Control group $(n=322)$} & \multicolumn{3}{|c|}{ Experimental group $(n=347)$} & \multirow{2}{*}{$\boldsymbol{P}$} \\
\hline & Mild to moderate & Severity & Extremely severe & Mild to moderate & Severity & Extremely severe & \\
\hline Accident $(\mathrm{n}, \%)$ & $79(27.5)$ & $102(35.5)$ & $106(36.9)$ & $52(17.6)$ & $77(26.0)$ & $167(56.4)$ & 0.000 \\
\hline Rescue (n,\%) & $41(23.6)$ & $63(36.2)$ & $70(40.2)$ & $26(12.9)$ & $53(26.4)$ & $122(60.7)$ & 0.000 \\
\hline Rescue success rate $(\mathrm{n}, \%)$ & $6(20.7)$ & $10(34.5)$ & $13(44.8)$ & $1(3.2)$ & $8(25.8)$ & $22(71.0)$ & 0.049 \\
\hline Hospital stay (d) & $21(15.8)$ & $44(33.1)$ & $68(51.1)$ & $13(8.2)$ & $36(22.6)$ & $110(69.2)$ & 0.006 \\
\hline
\end{tabular}




\subsection{The Modified MEWS Score Before and After Adjustment Predicts Clinical Outcome}

With death and ICU admission as the event endpoints, the correlation between the occurrence of the event and the MEWS score and the area under the ROC curve was statistically different between the two groups $(P<0.05)$. With death as the event endpoint, the modified MEWS score area under the ROC curve (AUC) of the experimental group after adjustment was 0.929 (95\% CI 0.882 0.976), and the best cut-off value corresponding to the Youden index was 10.5 points, and the best cut-off value was taken. The score is 11 , the sensitivity is $83.9 \%$, and the specificity is $83.9 \%$. The area under the ROC curve (AUC) of the modified MEWS score was 0.899 (95\% CI $0.834 \sim 0.965)$, the best cut-off value corresponding to the Youden index was 8.5 points, the best cut-off value was 9 points, the sensitivity was $72.4 \%$., and the specificity is $95.2 \%$. Taking ICU admission as the event end point, the modified MEWS score of the experimental group after adjustment was 0.680 (95\% CI $0.621 \sim 0.739)$, and the best cut-off value corresponding to the Youden index was 4.5 points, and the best cut-off was selected. The value is 5 points, the sensitivity is $60.2 \%$, and the specificity is $71.6 \%$. The area under the ROC curve (AUC) of the modified MEWS score was 0.902 (95\% CI $0.870 \sim 0.935)$, the best cut-off value corresponding to the Youden index was 6.5 points, the best cut-off value was 7 points, and the sensitivity was $86.2 \%$, the specificity is $81.6 \%$. The specific values are shown in Table 3 .

Table 3. The modified MEWS score before and after adjustment to predict clinical outcome.

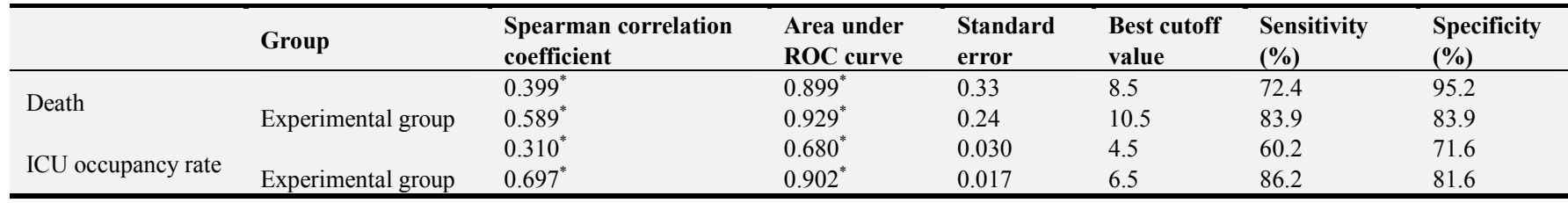

$\mathrm{P}<0.05$

\section{Discussion}

The MEWS scoring system has been widely used as an early warning tool for patients in internal medicine and emergency departments in recent years because it is easy to use, fast, and can scientifically and timely reflect the changes in the patients' condition. It can help nurses complete the risk prediction of the patient when the patient is admitted to the hospital, and classify the patients' risk according to the prediction result, so as to choose the corresponding nursing measures to reduce the mortality of the patient, reduce the length of stay, and reduce the ICU occupancy rate [9, 10]. At present, MEWS scoring has been widely used in the treatment of acute and severe patients, and relevant departments have gradually established a MEWS scoring system with disciplinary characteristics [11, 12]. However, studies have shown that the MEWS scoring system has only moderate predictive value in assessing the adverse clinical outcomes of patients [14], and the Department of Cardiology still lacks a MEWS scoring system with unique disciplinary characteristics. The purpose of this study is to analyze the application of the modified MEWS score after adjustment for effect in cardiovascular medicine patients, and to explore its clinical value and feasibility.

The analysis of the different clinical outcomes of the two groups of patients showed that the composition ratios of the two groups of the same grade were different. The incidence of adverse clinical effects in the experimental group was substantially lower than that in the control group, and the highly severe group had poor clinical outcomes. The incidence of outcome was substantially higher than that of the control group $(P<0.05)$, which indicates that the corrected MEWS score prediction accuracy rate was substantially better than that of the control group.

From the comparison of the evaluation results of the two scoring methods, it is shown that the sensitivity of the adjusted MEWS score is better than the MEWS score in predicting the death of cardiovascular disease patients, and the sensitivity and specificity are better than the MEWS score in predicting the ICU admission of cardiovascular disease patients. The differences were statistically significant $(P<0.05)$. This result indicates that the MEWS score has a moderate predictive value for the evaluation of patients' adverse clinical events, and the adjusted MEWS score has a high predictive value for the assessment of patients adverse clinical events. In this study, the best cut-off value of the MEWS score to evaluate the patients' condition is 5, which is similar to the results of Liling $\mathrm{Z}$ et al. [13] Tirotta, D et al. [14].

\section{Conclusion}

The corrected modified MEWS score further improves the accuracy of early warning based on the general modified MEWS score. It can be used to identify potentially critically ill patients.

\section{Funding}

2019 Zhuhai City Medical and Health Technology Plan Project;

Project Name: Construction and Application of Early Warning Care Work Model for Cardiovascular Diseases Based on the Corrected MEWS Scoring System; 
Project Number: 20191207A010021; Project Leader: Wu Juxian.

\section{References}

[1] Subbe C P, Krueger M, Gemmel L. Patients at risk: validation of early warning score in medical admissions [J]. BJA British Journal of Anaesthesia, 2001, 87 (2): 359P-360P.

[2] Shaikh M A, Punshi A, Talreja M L, et al. Comparison of within 7 Day All-Cause Mortality among HDU Patients with Modified Early Warning Score of $\geq 5$ with those with Score of $<5[J]$. Pakistan journal of medical sciences, 2021, 37 (2): 515-519.

[3] Roney J K, Whitley B E, Maples J C, et al. Modified early warning scoring (MEWS): evaluating the evidence for tool inclusion of sepsis screening criteria and impact on mortality and failure to rescue [J]. Journal of clinical nursing, 2015, 24 (23-24): 3343-3354.

[4] Jiang X, Jiang P, Mao Y. Performance of Modified Early Warning Score (MEWS) and Circulation, Respiration, Abdomen, Motor, and Speech (CRAMS) score in trauma severity and in-hospital mortality prediction in multiple trauma patients: a comparison study [J]. PeerJ, 2019, 7: e7227.

[5] Mitsunaga T, Hasegawa I, Uzura M, et al. Comparison of the National Early Warning Score (NEWS) and the Modified Early Warning Score (MEWS) for predicting admission and in-hospital mortality in elderly patients in the pre-hospital setting and in the emergency department [J]. PeerJ, 2019, 7: e6947.

[6] Yu Z, Xu F, Chen D. Predictive value of Modified Early Warning Score (MEWS) and Revised Trauma Score (RTS) for the short-term prognosis of emergency trauma patients: a retrospective study [J]. BMJ Open, 2021, 11 (3): e41882.
[7] Young K W, Jung S Y, Mi L J, et al. Modified Early Warning Score Changes Prior to Cardiac Arrest in General Wards [J]. Plos One, 2015, 10 (6): e0130523.

[8] Devoe B, Roth A, Maurer G, et al. Correlation of the predictive ability of early warning metrics and mortality for cardiac arrest patients receiving in-hospital Advanced Cardiovascular Life Support [J]. Heart \& Lung the Journal of Critical Care, 2016: 497-502.

[9] Yanmei W, Ping L, Min H, et al. Comparison of Modified Early Warning Score and Acute Physiology and Chronic Health Evaluation-II Score System in Evaluating the Ability of Patients in Emergency Internal Medicine [J]. Nurs J Chin PLA, 2017, 34 (22): 53-56.

[10] Lee Y J, Cho K J, Kwon O, et al. A multicentre validation study of the deep learning-based early warning score for predicting in-hospital cardiac arrest in patients admitted to general wards [J]. Resuscitation, 2021.

[11] Clemency B M, Murk W, Moore A, et al. The EMS Modified Early Warning Score (EMEWS): A Simple Count of Vital Signs as a Predictor of Out-of-Hospital Cardiac Arrests [J]. Prehosp Emerg Care, 2021: 1-22.

[12] Knoery C, Barlas R S, Vart P, et al. Modified early warning score and risk of mortality after acute stroke [J]. Clin Neurol Neurosurg, 2021, 202: 106547.

[13] Liling Z, Yuyan L, Luxiang Z, et al. Application Value of MEWS Score in Early Identification of Potentially Critical Patients in Emergency Department [J]. China Health Standard Management, 2021, 12 (3): 164-166.

[14] Tirotta D, Gambacorta M, La Regina M, et al. Evaluation of the threshold value for the modified early warning score (MEWS) in medical septic patients: a secondary analysis of an Italian multicentric prospective cohort (SNOOPII study) [J]. QJM, 2017, 110 (6): 369-373. 